\section{AURA3 trial: does Tagrisso (osimertinib) have the potential to become the new standard of care for second-line treatment of patients with EGFR T790M mutation-positive locally advanced or metastatic NSCLC}

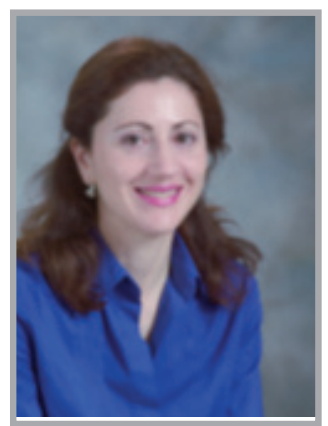

Vassiliki A Papadimitrakopoulou* speaks to Roshaine Wijayatunga, Managing Commissioning Editor: Dr Papadimitrakopoulou is the Jay and Lori Eisenberg Distinguished Professor of Medicine and Chief of the section of Thoracic Medical Oncology in the Department of Thoracic/Head and Neck Medical Oncology at the University of Texas/MD Anderson Cancer Center. Her areas of expertise include design and development of novel therapeutic clinical trials for lung and head and neck neoplasms, personalized genomics-driven lung cancer therapy and translational research and cancer chemoprevention. Her extensive experience in design, development and implementation of translational research in the context of multidisciplinary research teams has led to research funding from National Cancer Institute ( $\mathrm{NCl})$, American Society of Clinical Oncology (ASCO) and Department of Defense (DOD) both independently and as a member of a research team in the Head and Neck SPORE program. Currently, she serves as the principal investigator and leads numerous clinical and translational research projects with a focus on the development of biomarker-based targeted therapy to overcome therapeutic resistance in advanced disease and immunotherapy. Most notably, she has led the multidisciplinary clinical and translational research infrastructure dedicated to the treatment of metastatic refractory NSCLC as part of the BATTLE-2 program, designed and developed the first-in-the-world comprehensive genomics-driven umbrella approach in Squamous Lung Cancer, the Lung Master protocol, jointly sponsored by NCl-Cancer Therapy Evaluation Program (CTEP) and Foundation for the National Institutes of Health (FNIH)/industry, aiming at bringing personalized medicine to patients with this disease. She is the Co-PI of an R01 award focusing on the role of KRAS mutations and targeting in lung cancer. She is the lead author or coauthor of over 150 published articles, book chapters and reviews, and numerous abstracts involving cancer therapeutics, prevention and translational research and she has received several awards including the ASCO Young Investigator and Career Development Award. On this R01 application, she will serve as Co-PI, working closely with Roy Herbst (Yale Cancer Center) and Don Gibbons (UT/MD Anderson Cancer Center), building on the recently completed BATTLE-2 program, and capitalizing on both laboratory findings supporting MEK targeted therapy

*Thoracic Head \& Neck Medical Oncoology Department, University of Texas MD Anderson Cancer Center, TX, USA vpapadim@mdanderson.org

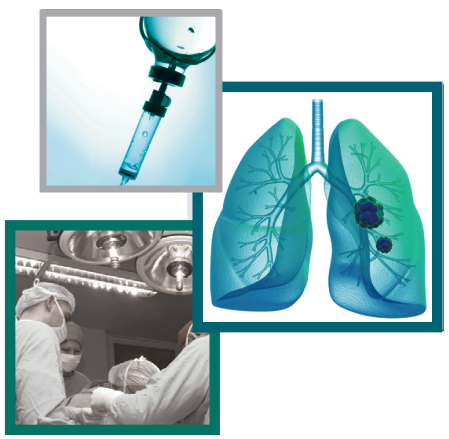


and clinical effectiveness of immunotherapy and their combinations in addressing KRAS mutated lung cancer.

First draft submitted: 11 January 2017; Accepted for publication: 2 February 2017; Published online: 6 March 2017

KEYWORDS

- EGFRT790M • NSCLC

- osimertinib
Q You are currently the Professor of Medicine in the Department of Thoracic/Head \& Neck Medical Oncology at the University of Texas MD Anderson. Can you tell our readers how your career has progressed to this point - how did you decide to specialize in lung, head \& neck oncology \& what has been your greatest achievement so far?

I have had an early interest in lung and head and neck malignancies through my medical oncology training and have now had 20 years' experience in research and treatment of these malignancies.

The most important achievements have been the development and execution of large, precision medicine, biopsy-driven umbrella clinical trials that serve not only as a means of improving therapeutic outcomes for patients but also as vehicles of discovery. Examples include the BATTLE trials (BATTLE-1 [1], BATTLE-2 [2]) and the National Clinical Trials Network (NCTN) supported Lung Master Protocol [3].

Q Given your extensive experience in this area - how important is the implementation of translational research \& multidisciplinary research teams to drug development in your opinion?

Extremely important and our efforts with the programs mentioned above are testament to their importance. It is only through careful selection of biomarkers that we can make progress in delivering precision medicine to patients and team science is absolutely essential in achieving this goal.

Q Can you tell our readers a little about the 'Lung Master Protocol': the 'first-in-the-world comprehensive genomics-driven umbrella approach in squamous lung cancers' that you designed \& developed?

We designed the Master Protocol for genomic screening and multi-sub-study testing of drug/ biomarker combinations in a Phase II/III setting compatible with subsequent US FDA approval. Sites participating in the National Cancer Institute (NCI) National Clinical Trials
Network provide a large patient resource, and this resource undergoes genomic screening. The screening is designed to identify a series of molecular targets or biomarkers for which there will be a new drug match. This then allows for the appropriate substudy assignment along with the correct drug treatment. Every molecular target included in S1400 will be represented by a biomarker for which there will be an analytically validated diagnostic assay. By taking this approach you have the basis for a largescale screening/clinical registration trial with the capacity to screen patients either through genomic analysis or immunohistochemistrybased assays, with homogeneous eligibility criteria and direct them to a substudy of the trial based on the results of screening diagnostic tests.

\section{Q What are the challenges associated} with the development of therapies for the treatment of NSCLC, \& particularly in mutation types \& even secondary mutations?

There are various challenges associated with drug development, including difficulties in patient recruitment to clinical trials, the many bureaucratic processes necessary for clinical trial initiation and the long periods of regulatory review. By modernizing this process with innovative study design that lowers costs and improves the current inefficiencies of the process of drug development, while hopefully identifying promising compounds in specific populations, a variety of stakeholders, along with ourselves, could begin the development and testing of innovative approaches for clinical trials - for example, the development of algorithms for clinical trial designs to facilitate early drug/biomarker codevelopment.

Q Can you please tell us a bit about the development of the third-generation drug osimertinib \& its mechanism of action? Osimertinib is an oral, irreversible, CNS active, EGFR-TKI selective for both EGFRm and T790M resistance mutations which occur in $50-60 \%$ of patients with progression after EGFR tyrosine kinase inhibitors which are the standard 
first-line therapy for patients whose tumors harbor the EGFR mutation. The development of osimertinib in the setting of T790M-positive tumors proceed through the classical steps of Phase I and Phase II studies that confirmed high response rate $(\sim 60 \%)$ and long progression-free survival (9.7-11 months). These findings led to accelerated approval of osimertinib.

Q What was the rationale behind the AURA3 trial? Can you tell us a bit about the study design (demographics, among others)?

AURA3 is a confirmatory trial required for full approval of osimertinib by the FDA and the EMA and it is therefore a randomized Phase III trial to demonstrate superiority of osimertinib over platinum-based doublet chemotherapy for patients with T790M-positive NSCLC.

Eligible patients had histologically or cytologically documented locally advanced or metastatic NSCLC and evidence of disease progression following first-line EGFR-TKI therapy. Documented EGFRm and central confirmation of tumor T790M mutation (using the cobas ${ }^{\circledR}$ EGFR Mutation Test [Roche Molecular Systems, CA, USA]) after first-line EGFR-TKI treatment was required. All patients were required to provide a blood sample at screening to test for T790M in plasma circulating tumor DNA. Patients with stable, asymptomatic CNS metastases, not requiring steroids for at least 4 weeks before first dose were eligible for inclusion.

Patients were stratified (Asian/nonAsian) and randomized 2:1 to receive oral osimertinib $80 \mathrm{mg}$ once daily or intravenous platinum pemetrexed (every 3 weeks for up to six cycles of pemetrexed $500 \mathrm{mg} / \mathrm{m}^{2}$ plus either carboplatin AUC5 or cisplatin $75 \mathrm{mg} / \mathrm{m}^{2}$ ). Patients whose disease had not progressed after four cycles of platinum pemetrexed could continue maintenance pemetrexed according to the approved label and in adherence with FDA-approved toxicity management guidelines or, where appropriate, local approved or clinical practice guidelines.

\section{Q The data from the AURA3 trial were presented recently at the 17th World} Conference on Lung Cancer in Vienna. Can you tell our readers about the study results? The primary outcome of progression-free survival (PFS) was statistically and significantly longer in the osimertinib group than in the platinum-pemetrexed group: HR: 0.30 (95\% CI: 0.23-0.41); $\mathrm{p}<0.001$ (median 10.1 vs 4.4 months).
Overall response rate was significantly higher in the osimertinib group than in the platinumpemetrexed group (71\% [95\% CI: 65-76] vs 31\% [95\% CI: 24-40]; OR: 5.39 [95\% CI: 3.47-8.48]; $\mathrm{p}<0.001)$.

Q There were particularly interesting results in patients with CNS metastases. What are the challenges associated with the treatment of lung cancer patients with brain metastases \& what was the rationale in using osimertinib to treat these particular patients?

Osimertinib resulted in a $68 \%$ reduction in risk of disease progression or death in the absence of progression compared with chemotherapy in all predefined subgroups analyzed, including patients with CNS metastases (HR: 0.32 [95\% CI: 0.21-0.49]; median PFS 8.5 vs 4.2 months).

This is particularly important for patients who present with brain metastatic disease, which can become rapidly debilitating and carries an adverse prognosis for overall survival. Although advances have been made in more extensive use of stereotactic radiation therapy for small volume disease, a great number of patients only benefits from radiation therapy for a limited time making the development of CNS active drugs extremely important.

Q What is the significance of the AURA3 trial results? Will this lead to a change in standard of care?

These results establish osimertinib as the standard of care therapy for EGFR mutation-positive patients whose tumor develops a T790M mutation as the mechanism of resistance and who progress on first-line EGFR-TKI therapy.

\section{Q What are the next steps for research into} osimertinib?

Questions that are currently being explored are the use of osimertinib in the first-line treatment of patients with EGFR mutation-positive tumors, the prevention of resistance to osimertinib through combination therapy and the description and successful targeting of mechanisms of resistance to osimertinib.

Q How would you like to see this area of research develop over the next 5-10 years? All the questions outlined above will be hopefully answered; in addition the role of osimertinib and other inhibitors in early disease stages 
treated with either surgical resection and or radiation therapy will be defined. Eventually we hope that research in this setting will allow patients with EGFR mutation-positive NSCLC to live longer and also allow more patients with the disease to be offered curative therapy.

Q Do you have any closing remarks for our readers?

With the results of AURA3, it becomes extremely important for all practitioners to provide testing for the T790M to their patients progressing on first-line EGFR-TKIs to allow enhanced access to the new standard of care therapy to all patients.

\section{Disclaimer}

The opinions expressed in this interview are those of the interviewee and do not necessarily reflect the views of Future Medicine Ltd.

\section{Financial \& competing interests disclosure}

VA Papadimitrakopoulou has an Astra Zeneca advisory role, research finding to institution. VA Papadimitrakopoulou has no other relevant affiliations or financial involvement with any organization or entity with a financial interest in or financial conflict with the subject matter or materials discussed in the manuscript apart from those disclosed.

No writing assistance was utilized in the production of this manuscript.

\section{References}

1 Kim ES, Herbst RS, Wistuba II et al. The BATTLE trial: personalizing therapy for lung cancer. Cancer Discov. 1(1), 44-53 (2011). et al. The BATTLE-2 study: a biomarker-

integrated targeted therapy study in previously treated patients with advanced non-small-cell lung cancer. J. Clin. Oncol. 34(30), 3638-3647 (2016).

3 Herbst RS, Gandara DR, Hirsch FR et al. Lung Master Protocol (Lung-MAP) - a biomarker-driven protocol for accelerating development of therapies for squamous cell lung cancer: SWOG S1400. Clin. Cancer Res. 21(7), 1514-1524 (2015). 